\title{
Hemorragia de tubo digestivo alto no varicial, ¿hay algo nuevo en nuestro arsenal?
}

\author{
Juan M. Abdo-Francis* \\ Departamento de Gastroenterología, Hospital Ángeles Acoxpa, Ciudad de México, México
}

La hemorragia de tubo digestivo alto no varicial (HTDANV) es la principal causa de atención de urgencias y hospitalización por enfermedad gastrointestinal a nivel mundial, con una incidencia aproximada de entre 40 y 150 casos por 100,000 habitantes'. A pesar de que en los últimos años su mortalidad ha disminuido de manera importante, la HTDANV sigue representando un reto tanto diagnóstico como terapéutico. En la Digestive Disease Week 2020 y en la United European Gastroenterology Week 2019 se presentaron numerosos trabajos en los que muestran los principales adelantos para el manejo de los pacientes.

Los trabajos presentados en el DDW 2020 se centraron en el momento adecuado para la realización de la endoscopía en el paciente con hemorragia activa, tema de controversia en la comunidad médica mundial, y en el uso de técnicas conocidas que cada vez están más al acceso de los médicos de países en vías de desarrollo, como el hemospray y el uso de ácido tranexámico. A su vez, se consideraron las diversas escalas pronósticas y se comparó su capacidad predictiva. En un estudio prospectivo realizado en Tailandia por el Dr. Chang y sus colaboradores evaluaron las escalas de AIMS65, Glasgow-Blatchford y Rockall con el objetivo de encontrar la escala más adecuada para pacientes con hemorragia de tubo digestivo alto, tanto varicial como no varicial. Sus resultados mostraron que las tres escalas son buenas predictoras para la sobrevida del paciente, mientras que Glasgow-Blatchford mostró también resultados estadísticamente significativos para necesidad de transfusión ${ }^{2}$. Dentro de los aditamentos utilizados en los trabajos presentados, el hemospray fue el más empleado debido a su mayor accesibilidad a nivel mundial. Los doctores miembros del Registro Internacional de Uso de Hemospray presentaron un estudio en que se evaluaron parámetros de sobrevida de 500 pacientes en 16 centros, tanto europeos como de Estados Unidos. Los pacientes tuvieron como origen de sangrado úlcera péptica (46\%), malignidad (19\%), esofagitis/gastritis/duodenitis (4\%), desgarro de Mallory-Weiss (3\%) y angiodisplasia (2\%). Reportaron que en HTDANV hubo hemostasia inmediata en pacientes en los que se utilizó, ya sea como monoterapia $(95 \%)$, terapia combinada $(91 \%)$ o terapia de rescate $(90 \%)$. Destacan también que la mayor efectividad se alcanza en pacientes post endoterapia, malignidad y angiodisplasia. A su vez, mencionan que tuvieron fracaso en el tratamiento en $7.8 \%$ de los pacientes, siendo la mayoría de ellos de origen por úlcera péptica ${ }^{3}$. Por su parte, el Grupo encabezado por el Dr. Becq de la División de Endoscopía del Saint Antoine Hospital en Boston, valoró el uso de hemospray en pacientes que requieren abordaje de urgencia. Se evaluaron 152 pacientes y a 31 endoscopistas de diferentes grados de experiencia. La sobrevida a 30 días fue de $71.6 \%$ y solo se reportó 1 complicación (perforación), concluyendo que el abordaje de urgencia, inclusive en horarios no laborales por endoscopistas de guardia, tienen 
resultados satisfactorios ${ }^{4}$. Finalmente, el Dr. Bazarbashi y sus colaboradores presentaron un análisis del uso de hemospray en 20 pacientes con hemorragia asociada a malignidad, mostrando una efectividad en la detención del sangrado del $90 \%$ y sugiriendo que la hemostasia puede ser prolongada para pacientes no candidatos a otra terapia al momento del manej $0^{5}$.

La Dra. Twum-Barimah y sus colaboradores presentaron una revisión sistemática y metaanálisis de estudios aleatorizados usando ácido tranexámico para HTDANV. Se evaluaron 2013 pacientes distribuidos en 10 estudios en el que se evaluó el ácido tranexámico contra placebo, reportando que los estudios muestran evidencia moderada de la efectividad del ácido vs. placebo, en especial en reducción de la mortalidad en pacientes con hemorragia activa ${ }^{6}$. A su vez, el Dr. Alzahrani y los investigadores de la Universidad McMaster de Canadá presentaron un metaanálisis en que se evaluó el uso de somatostatina y sus análogos versus el uso de antagonistas $\mathrm{H} 2$ e inhibidores de la bomba de protones en la hemorragia no varicial. Se evaluaron 26 estudios, con un total de 1912 pacientes. Sin embargo los estudios eran de calidad moderada a baja por lo que solo se pudo establecer el efecto en de la somatostatina contra monoterapia con IBP, mientras que el efecto en mortalidad, necesidad de cirugía y riesgo de resangrado no pudo ser evaluado ${ }^{7}$.

En la UEGW 2019 se presentaron varios trabajos que, a diferencia del DDW, abordan herramientas alternativas al hemospray como el UI-EWD y el uso de clips, así como el uso de anticoagulantes previo al manejo endoscópico de pacientes con hemorragia de tubo digestivo alto. Los Dres. Robles y Alcívar de Ecuador presentaron un estudio de casos controles comparando el uso de clips OVESCO ${ }^{\text {TM }}$ como primera línea de tratamiento en pacientes con úlcera péptica con alto riesgo de sangrado. De 95 pacientes, a 46 se les aplicó OVESCO ${ }^{\text {TM }}$ y al resto hemoclips, reportando menor resangrado a las 48 horas con menor tiempo de procedimiento ${ }^{8}$. El grupo encabezado por el Dr. Lan del West China Hospital evaluaron el uso de embolización profiláctica por angiografía transcatéter con resultados positivos para evitar resangrado (3.7\% vs.
$25.6 \%$ ) en 86 pacientes con Forrest $I I A^{9}$. Un estudio presentado por investigadores del Hospital de la Universidad Inha en Corea del Sur, evaluó el uso de un nuevo polvo hemostático contenido en un apósito endoscópico para heridas aplicado en 56 pacientes con HTDANV con resultados prometedores en el $96.4 \%$ de los pacientes ${ }^{10}$.

\section{Conflicto de intereses}

Los autores declaran no tener conflicto de intereses alguno.

\section{Financiamiento}

Los autores no recibieron patrocinio para llevar a cabo este artículo.

\section{Bibliografía}

1. Van Leerdam ME. Epidemiology of acute upper gastrointestinal bleeding. Best Pract Res Clin Gastroenterol 2008; 22: 209-224

2. Arunchai Chang, Chokethawee Ouejiraphant, et al. Comparison Of The Aims65, Glasgow-blatchford Score And Rockall Score For Predicting Outcomes In Patients With Nonvariceal And Variceal Upper Gastrointestinal Bleeding: A Prospective Single Center Study. Sesión de carteles presentada en: DDW; 2020 mayo 2-5; Chicago, IL. Su1404-2020

3. Mohamed Hussein, Durayd Alzoubaidi, et al. Hemospray In The Treatment Of Non-variceal Upper Gastrointestinal Bleeds: Outcomes From The First 500 Patients From The International Hemospray Registry. Sesión de carteles presentada en: DDW; 2020 mayo 2-5; Chicago, IL. 963-2020

4. Aymeric Becq, Charles Houdeville, et al. Real Life Experience With The Use Of A Hemostatic Powder In 152 Patients Undergoing Urgent Endoscopy For Gastrointestinal Bleeding. Sesión de carteles presentada en: DDW; 2020 mayo 2-5; Chicago, IL. Tu1494-2020.

5. Ahmad Najdat Bbazarbashi, Lolwa Al Obaid, et al. Endoscopic Hemostatic Powder For The Treatment Of Malignancy-related Gastrointestinal Bleeding: A Single Center Us Experience. Sesión de carteles presentada en: DDW; 2020 mayo 2-5; Chicago, IL. 499-2020.

6. Erica Twum-Barimah, Ibtihal S. Abdelgadi, et al. Tranexamic Acid For Upper Gastrointestinal Bleeding: A Systematic Review And Meta-analysis Of Randomized Controlled Trials. Sesión de carteles presentada en: DDW; 2020 mayo 2-5; Chicago, IL. Su1410-2020.

7. Ibrahim M. Alzahrani, Kristyne Onizuk, et al. Somatostatin And Analogues For Acute Non-variceal Upper Gastrointestinal Bleeding: A Systematic Review And Meta-analysis Of Randomized Trials. Sesión de carteles presentada en: DDW; 2020 mayo 2-5; Chicago, IL. Mo1010-2020.

8. Robles-Medranda C. Alcivar-Vasquez J, et al. Over-the-Scope Clip as First-Line Therapy in the Management of High.risk bleeding Peptic UIcers: A Case-Match Control Study. Sesión de carteles presentada en: UEGW; 2019 octubre 21-23; Barcelona, IL. P0546-2020.

9. Lan T, Tong H, Wu H, Tang C. P. Prophylactic Transcatheter Angiographic Embolization Reduces the Risk of Rebleeding in Patients with Forres IIA Ulcer: A Retrospective Study. Sesión de carteles presentada en: UEGW; 2019 octubre 21-23; Barcelona, IL. P0544-2020.

10. Park J-S, Lee D-H, et al. Novel Hemostatic Adhesive Powder Application in Nonvariceal Upper Gastrointestinal Bleeding. Sesión de carteles presentada en: UEGW; 2019 octubre 21-23; Barcelona, IL. OP248-2020. 